\title{
¿PUEDE EL CONOCIMIENTO SER UN ESTADO MENTAL?
}

\author{
FLORENCIA RIMOLDI \\ Universidad de Buenos Aires - CONICET \\ rimoldiflorencia@hotmail.com
}

\section{Resumen}

En Knowledge and its Limits, Timothy Williamson argumenta a favor de la tesis fuerte de que el conocimiento es un estado mental (CEMf), ofreciendo una caracterización del conocimiento según la cual es "la actitud factiva estativa más general". Dicha caracterización es central una vez que se considera que los estados perceptivos son también actitudes factivas estativas. Esta propuesta ha sido discutida ampliamente en la literatura. En este trabajo argumento en contra de (CEMf) a partir de una crítica novedosa que parte de las herramientas mismas que Williamson usa a su favor. Para ello argumentaré, en distintas etapas, que los estados perceptivos no son actitudes factivas estativas. La crítica en cuestión no se limita a mostrar la falsedad de la caracterización del conocimiento en términos de actitudes factivas estativas, sino que también muestra la implausibilidad de (CEMf).

PALABRAS CLAVE: Factividad; Clases naturales; Actitudes; Acción.

\begin{abstract}
In Knowledge and its Limits, Timothy Williamson defends the strong thesis that knowledge is a mental state (CEMf), while offering a characterization of knowledge according to which it is the "most general factive stative attitude". This characterization becomes central to the Project once we assume that perceptual states are also factive stative attitudes. This proposal has been widely discussed in the literature. In this paper I propose a novel objection against (CEMf) by using the very same tools that Williamson deploys on behalf of his view. I will argue, in different stages, that perceptual states are not factive stative attitudes. My criticism is not limited to showing that the characterization of knowledge in terms of factive stative attitudes is false, it also shows that (CEMf) is implausible.
\end{abstract}

KEY WORDS: Factiveness; Natural Types; Attitudes; Action.

\section{El conocimiento como estado mental}

En este trabajo me ocupo de la tesis sobre la cual se organiza el proyecto epistemológico de Timothy Williamson (2000). Esa tesis es que el conocimiento es un estado mental, y tiene dos lecturas. Según la primera lectura, trivialmente verdadera, la tesis captura la idea de que para que un sujeto sepa algo, es necesario que su mente esté en cierto 
estado. Según la otra lectura, más controvertida, la condición de que la mente esté en cierto estado no solo es necesaria sino suficiente. Esta es la tesis central de Williamson $(2000, \text { p. } 20)^{1}$ :

\section{(CEMf) Para algún estado mental $S$, estar en $S$ es necesario $y$ suficiente para conocer que $p$.}

Para Williamson, el conocimiento es un estado mental en el sentido en que creer, amar y desear, entre otros, lo son. Es decir, el conocimiento es un genuino estado mental. Es importante aclarar que un estado mental genuino no es un estado mental complejo (compuesto por estados mentales simples) ni necesita formar parte de otros estados mentales para ser el estado mental que es. Más todavía, como mostraré más adelante (sección 3) la teoría misma de Williamson presenta al conocimiento como un estado mental con eficacia causal. Por estas razones, Williamson está comprometido con una particular teoría sobre la metafísica del conocimiento como estado mental. ${ }^{2} \mathrm{Su}$ concepción específica del conocimiento hace prevalecer una comprensión naturalista del conocimiento, frente a una normativista o de tipo social. Desde esta perspectiva, si el conocimiento es un estado mental genuino, entonces el fenómeno de conocer es una clase natural y, como tal, debe ser estudiado no por la epistemología (con su pretensión de explicar la naturaleza del conocimiento o al menos la diferencia entre conocimiento y mera creencia verdadera) sino por la psicología, la neurobiología o, como mucho, por la filosofía de la mente ${ }^{3}$.

${ }^{1}$ Las traducciones de los textos citados en este trabajo son mías.

${ }^{2}$ Ciertamente, la postura de Williamson es incompatible tanto con un eliminativismo como con un dualismo sobre la metafísica de los estados mentales. El eliminativismo sostiene que los estados mentales son clases de la psicología común que no encuentran un lugar en una psicología rigurosa y que, por ende, deben eliminarse. Una vez eliminado, el conocimiento como estado mental no solo no sería un estado mental genuino sino que tampoco tendría eficacia causal. El dualismo, por su parte, sostiene que los estados mentales son clases no naturales de naturaleza sui géneris, distinta, por ejemplo, a la de los estados y fenómenos físicos. Es bien conocido que uno de los graves problemas de esta postura es su incapacidad por dar cuenta de la eficacia causal de los estados mentales. Si los estados mentales no son estados naturales, simplemente no se entiende cómo es que pueden ejercer influencia causal sobre objetos naturales en el mundo. De ahí que Williamson tampoco pueda aceptar una metafísica dualista sobre la naturaleza del conocimiento como estado mental genuino.

${ }^{3}$ Es importante aquí distinguir entre dos afirmaciones condicionales. Según la primera, si el conocimiento es un estado mental genuino, entonces constituye una clase natural. La segunda sostiene que si algo es un estado mental entonces constituye una clase natural. Esta última es una afirmación más general que no pretendo discutir aquí ni tiene relevancia para la discusión presente. Lo que está en juego en este trabajo es 
Ahora bien, el conocimiento es factivo. Esto significa que solo se puede tener conocimiento de proposiciones verdaderas. La existencia de estados mentales que presentan tal característica de infalibilidad puede llamar la atención como algo en principio contraintuitivo, en la medida en que la verdad de una proposición depende del entorno y no de la mente de un individuo. Williamson argumenta contra esto que esta concepción del conocimiento y otros estados factivos como estados mentales está en verdad incluida en nuestra idea preteórica de lo mental. En sus palabras:

Sería extraño si hubiera un estado mental de temer pero no de arrepentirse, o un estado mental de imaginar pero no uno de recordar. De hecho, no es claro que haya bases preteóricas para omitir a las actitudes factivas de la lista de estados mentales paradigmáticos. La idea de que lo mental incluye conocer y otras actitudes factivas forma parte de la comprensión natural del procedimiento por el cual adquirimos el concepto de lo mental. Por supuesto, eso no excluye el subsecuente descubrimiento de razones teóricas para trazar la línea entre lo mental y lo no mental en otro lugar. Pero más vale que la teoría detrás de estas razones sea una buena teoría (2000, p. 22).

Si está en lo correcto, la plausibilidad prima facie de (CEMf) está supeditada a la ausencia de objeciones teóricas relevantes contra la misma $^{4}$. A lo largo de la primera parte de su libro, Williamson defiende (CEMf) frente a tres objeciones teóricas - la de la transparencia de los estados mentales, la de la irrelevancia causal del conocimiento y la de la independencia de los estados mentales a su entorno- tras lo cual considera allanado el terreno hacia una caracterización teóricamente sustantiva del conocimiento como estado mental. Este proceder está justificado en tanto lo correcto desde el punto de vista metodológico es respetar aquello que prima facie se presenta como correcto y supera exitosamente objeciones teóricas que podrían derrotarlo. La caracterización teórica en cuestión es que el conocimiento es la actitud factiva estativa más general.

la verdad de la primera afirmación en relación con las tesis defendidas por Williamson. En particular, se trata de la relación entre la consideración del conocimiento como estado mental genuino y su presencia ineliminable en explicaciones causales de la acción. No obstante, en la discusión contemporánea algunos autores dan por hecho el segundo condicional (Hellie 2007 y Kumar 2014).

${ }^{4}$ Se ha argumentado que de hecho la posición preteórica es demasiado vaga como para implicar la tesis de que los estados factivos son "estados mentales genuinos", es decir el externalismo de actitudes. Véase Cassam (2009) para un desarrollo de esta idea. 
La mayor parte de las críticas en torno a la tesis (CEMf) posteriores a la publicación de Knowledge and its Limits están centradas en la defensa de alguno de los tres puntos que Williamson presenta como objeciones a (CEMf), a partir de nuevos argumentos, o de críticas a los argumentos en contra de esos puntos ofrecidos en el libro. ${ }^{5}$ No es mi intención participar de ese debate, y considero que no es necesario hacerlo para mostrar la implausibilidad de (CEMf). Por el contrario, presentaré una cuarta objeción novedosa que parte de las herramientas mismas que Williamson usa a su favor y que se construye con los siguientes elementos. Primero, mostraré que hay buenas razones para pensar que el conocimiento no es una clase natural y, por ende, un estado mental. Sin buenas razones para defender lo anterior, la plausibilidad de (CEMf) dependerá únicamente de las virtudes teóricas de la caracterización sustantiva en cuestión. De ahí que, en un segundo momento, argumentaré que la propuesta carece de esas virtudes.

En lo que resta de esta sección desarrollaré la primera parte de mi objeción, tras lo cual debería ser claro cómo es que el fracaso de la propuesta específica de Williamson redundaría en un rechazo de la tesis general de que el conocimiento es un estado mental. En la segunda y tercera secciones presentaré la segunda parte de mi objeción.

\subsection{Razones en contra}

Si se considera que el fenómeno al que nos referimos cuando usamos el término "conocimiento" es un tipo de entidad, entonces es o bien natural, o bien social, o bien artificial ${ }^{6}$. Como ya se ha dicho, (CEMf) implica que el conocimiento es una clase natural. Creo que hay buenas razones para afirmar que aquello a lo que llamamos "conocimiento" no es una clase natural. ${ }^{7}$

Nuestras prácticas atributivas de conocimiento sugieren que atribuimos conocimiento a sujetos que se encuentran vinculados por un

${ }^{5}$ Véase Leite (2005), Fricker (2009), Neta y Rohrbaugh (2004), Steup (2009), Smithies (2012), entre muchos otros.

${ }^{6}$ Estas tres alternativas surgen de una consideración intuitiva acerca de los tipos de clases posibles que no constituyen meros agregados arbitrarios (véase, por ejemplo, Kusch 2002), pero no me comprometo fuertemente con la idea de que forman entre ellas una clasificación exhaustiva de tipos de entidades. Por otro lado, no es necesario comprometerse con ninguna de las tres opciones. Williams (2007) argumenta que no es posible construir una teoría del conocimiento precisamente porque el mismo no constituye ningún tipo de entidad.

${ }^{7}$ De hecho, en algunos autores como Hazlett (2010) y Kusch (2002) esto se da por sentado sin argumentación. 
conjunto de normas sociales, a diferencia de, por ejemplo, nuestras prácticas atributivas sobre ser un tigre, tener apendicitis o ser una flor. Se atribuye a quien conoce una capacidad de dar razones a favor del pretendido conocimiento, una obligación de defender su verdad frente a objeciones, una habilitación a usarlo como premisa en ulteriores razonamientos, y un carácter de garante de ese conocimiento frente a quienes dicho conocimiento es manifestado. Autores como Austin (1961), Sellars (1956), Chisholm (1976), Welbourne (1993), Craig (1990) consideran que la existencia misma de estas prácticas de atribución de conocimiento son buenas razones para pensar que el estatus de conocimiento depende de una serie de relaciones sociales que exhiben su carácter no natural. Si bien este es un punto controvertido que merece su propia discusión, hacerlo va más allá de los límites del presente trabajo. Baste con señalar que, de aceptarse estas consideraciones, el fenómeno del conocimiento excedería los límites de los fenómenos puramente cognitivos -que bien pueden constituir clases naturales- del mismo modo en que, por ejemplo, el fenómeno de género excede a las categorías naturales sexuales.

No intento mostrar que el conocimiento sea de hecho un tipo social. Tan solo pongo en consideración una serie de características de las prácticas de atribución de conocimiento que son prima facie evidencia a favor de que el conocimiento no es una clase natural, y que cualquier explicación del conocimiento que no las recoja debe ocuparse de derrotar o explicar de una manera alternativa. Hay muchas formas de hacer esto. Quien defiende que el conocimiento es una clase natural puede lidiar con la supuesta evidencia considerándola sencillamente inadecuada. Estamos -diría un defensor de esa posición- simplemente equivocados cuando atribuimos esas características autoritativas y vinculantes a quienes conocen. Cuando se defiende que un fenómeno es natural, parte de lo que se afirma es que la ciencia debe ser la encargada de estudiar su naturaleza. De este modo, las prácticas atributivas no tienen un estatus evidencial fuerte, solo sirven para identificar un conjunto de casos paradigmáticos que permiten que la ciencia pueda recortar el fenómeno a estudiar (Haslanger 2006).

Ahora bien, en orden a poder seguir esa estrategia, el defensor de la propuesta naturalista debe mostrar primero que sus virtudes teóricas justifican la consideración del conocimiento como clase natural. Esto de mínima pone en pie de igualdad las consideraciones sobre nuestra noción preteórica de la mente a las que apela Williamson a favor de (CEMf) con aquellas originadas en nuestras prácticas atributivas y que apuntan en dirección contraria, haciendo de las 
virtudes teóricas de su propuesta el eje central para evaluar su plausibilidad. ${ }^{8}$

Podemos hallar en Williamson una defensa de (CEMf) a partir de dos virtudes teóricas de su propuesta sustantiva que entiende al conocimiento como un estado mental que constituye la actitud factiva estativa más general. (1) La primera virtud consiste en satisfacer la necesidad de postular un estado mental de conocer para explicar causalmente una serie de acciones que de otro modo no podrían explicarse adecuadamente. (2) La segunda virtud es la de ofrecer una respuesta al problema del valor del conocimiento a partir de su caracterización teórica.

En la segunda y tercera secciones lidiaré respectivamente con ambas cuestiones. En la segunda sección muestro que la propuesta es incapaz de dar cuenta del valor del conocimiento. En la tercera sección mostraré que no hay necesidad de postular estados mentales como conocer para explicar causalmente ninguna acción. En la cuarta sección muestro la inadecuación de una posible respuesta de Williamson.

\section{La caracterización sustantiva del conocimiento como estado mental}

En esta sección presentaré argumentos que sugieren que la tesis del conocimiento como la actitud estativa factiva más general no explica adecuadamente el valor del conocimiento. Estos argumentos son de tipo lingüístico y filosófico. Los primeros se basan en los esquemas temporales de Vendler. Esto es importante ya que el propio Williamson los utiliza para defender que "conocer" es un operador factivo de estados mentales o FMSO (factive mental state operator). Mis argumentos muestran que los términos de estados perceptivos no son FMSO. Los segundos se basan en evidencia de distinto tipo para mostrar que los estados perceptivos y de memoria no son estados factivos estativos.

\subsection{Los argumentos lingüísticos}

Williamson (2000, pp. 33-41) basa su caracterización del conocimiento en las propiedades lingüísticas del término "conocer" y otros términos que refieren a lo que comúnmente se considera que son las

\footnotetext{
${ }^{8}$ De máxima, si, tal como argumenta Cassam, esas consideraciones preteóricas no son concluyentes, entonces la necesidad de sopesar con virtudes teóricas un punto de partida implausible se torna aún más imperiosa.
} 
fuentes del conocimiento (percepción, memoria y razón). Postula, a su vez, la categoría lingüística de los FMSO, tomando como base los esquemas temporales postulados por Vendler (1967). Los FMSO son la realización, en un lenguaje natural, de las actitudes factivas estativas. Estas últimas son actitudes proposicionales que se diferencian de otras actitudes porque si uno tiene una de ellas hacia $p$, entonces necesariamente $\mathrm{p}$ es verdadera; porque son estados, no procesos; y porque no son descomponibles en elementos más simples. De este modo, amar, creer que $\mathrm{p}$, olvidar que $\mathrm{p}$, y creer con verdad que $\mathrm{p}$ respectivamente, no están dentro de esa categoría. Amar no es una actitud proposicional, creer que $\mathrm{p}$ no es una actitud factiva, olvidar que $\mathrm{p}$ no es un estado sino un proceso y creer con verdad que p es descomponible en los elementos más simples de creencia y verdad. Según Williamson, recordar, oír, ver, en cambio, sí lo están. La categoría de los FMSO es presentada como siendo "la expresión característica en el lenguaje de [las] actitud[es] factiva[s] estativa[s]" (2000, p. 34), es decir, como si los FMSO fueran términos que reflejan una clase de actitudes que se admiten como legítimamente existentes. $^{9}$

Son cinco sus características definitorias ${ }^{10-11}$ :

- Son inanalizables semánticamente. Según Williamson, esto equivale a no ser sinónimas con ninguna expresión compleja cuyo significado esté compuesto por el significado de sus partes. (2000, p. 34) Esta característica es compatible con la analizabilidad sintáctica, y puede marcar diferencias entre términos como "sentir" y "pudo sentir". En varios

${ }^{9}$ Lo cierto es que en un movimiento solapado Williamson hace que esas actitudes se modelen en virtud de los FMSO, y no a la inversa. La supuesta inanalizabilidad semántica de los FMSO debería estar reflejando el carácter no composicional de las actitudes factivas estativas, pero en verdad no hay razones independientes para considerar que a esa clase (si realmente es una clase existente) no puedan pertenecer actitudes compuestas como, por ejemplo, la de creencia verdadera justificada.

${ }^{10}$ Aunque en todo lo que sigue utilizaré el español como lenguaje a estudiar, he intentado usar solo expresiones que posean un correlato más bien directo en el inglés. Como Williamson observa, la categoría de los FMSO se realiza en otros idiomas, y el español parece ser uno de ellos. Cuando lo considere necesario anotaré al costado de la expresión en español la correspondiente en inglés en itálica, para que el lector evalúe la corrección de la traducción.

${ }^{11}$ Los ejemplos del (1) al (14) son una traducción de los ejemplos que Williamson mismo utiliza para caracterizar a los FMSO. Estos ejemplos no pretenden presentar evidencia acerca de los términos empleados. De ahí que, en principio, la fuerza argumentativa de los ejemplos es independiente de si se presentan en inglés o en español. De cualquier forma, en mi crítica a Williamson asumiré que los ejemplos son exitosos. 
idiomas la adición del auxiliar "puede" frecuentemente formaría un FMSO. ${ }^{12}$ De este modo "sintió", como en

(1) Ella sintió que el hueso estaba roto.

no expresaría según Williamson un FMSO, porque su lectura saliente es "ella intuitivamente creyó que el hueso estaba roto", donde "sintió" no es factivo (2000, p. 36). Mientras que "pudo sentir", en

(2) Ella pudo sentir que el hueso estaba roto.

sí lo es, pues la paráfrasis de su lectura saliente es "Ella supo mediante el sentido del tacto que el hueso estaba roto", donde "pudo sentir" es perceptual y factivo. Su analizabilidad sintáctica no es problema, mientras la expresión no sea analizable semánticamente.

"Creer con verdad", en cambio, no es un FMSO pues podemos descomponer al concepto de cree con verdad en los componentes de creencia y verdad.

- Los FMSO son factivos.

"Vio" y "pudo ver" serían ambos FMSO, pues serían ambos factivos. Aunque, según Williamson, solo el segundo posee un carácter más específicamente perceptual:

(3) Vio que no había nada de qué preocuparse.

(4) Pudo ver que el hombre llevaba un cuchillo.

Williamson observa que mientras "pudo oír" es factivo y semánticamente inanalizable (aunque puede ser parafraseado por "supo mediante el sentido del oído"), y por lo tanto es un FMSO, "oyó” no lo es, porque no es factivo:

(5) Ella oyó que el volcán estaba en erupción.

puede querer decir que ella oyó a alguien decir que el volcán estaba en erupción, y eso es compatible con la falsedad de lo dicho.

12 Williamson se hace eco de Vendler para sostener esta afirmación, pero Vendler no tenía en cuenta la existencia de los FMSO. Como se ha dicho, esa categoría es postulada por Williamson. 
La relación de implicación deductiva que, según Williamson, se da entre lo términos factivos y la proposición que les sigue no debe confundirse con la relación de presuposición. ${ }^{13}$ Williamson utiliza como ejemplo el término "adivinar" (guess). Hay un uso del verbo "adivinar" en el que "S adivinó que A" en cierto sentido presupone "A". Sin embargo, esta presuposición es cancelable por contexto, como la corrección lingüística y lógica de las siguientes oraciones muestra:

(6) Adiviné incorrectamente que él era culpable (I guessed incorrectly that he was guilty)

(7) Adiviné que él era culpable y tú adivinaste que él era inocente (I guessed that he was guilty and you guessed that he was innocent)

En contraste, la sustitución de "supe" por "adiviné" en (6) y (7) lleva a una contradicción (2000, p. 35).

- Los FMSO denotan actitudes proposicionales.

(8) María es responsable de que la bomba haya estallado.

"Ser responsable de" no es un FMSO porque no implica que María posea una actitud proposicional hacia la proposición en cuestión.

- Los FMSO denotan estados.

Aquí Williamson recoge los esquemas de tiempo de Vendler, dentro de los cuales se hallan los términos de estado. Los FMSO caen en ese conjunto. Los términos de estado poseen marcas lingüísticas que pueden ayudarnos a ver cuándo estamos en presencia de un FMSO. Por ejemplo, los tiempos continuos no pueden usarse con propiedad en casos de estado:

(9) Está probando que hay infinitos números primos.

(10) Los zapatos le están doliendo.

(11) Está sabiendo que hay infinitos números primos. *

(12) Está creyendo que hay infinitos números primos. *

(13) Los zapatos le están cabiendo. *

${ }^{13}$ Williamson no ahonda en ningún detalle respecto del tipo de presuposición en el que está pensando, de modo que no me detendré en eso en este trabajo. Sin embargo la caracterización de presuposición pragmática de Stalnaker (1974) podría completar el vacío. 
Las últimas tres oraciones son impropias. Esto es así porque "caber", "conocer" y "creer" son estativos, mientras que "doler" y "probar" no lo son.

Otros verbos pueden tener lecturas tanto estativas como no estativas:

(14) Ella está recordando que hay infinitos números primos.

En la lectura saliente de "recordar" -observa Williamson-, (14) es impropia, pero puede ser correctamente usada para decir que está en el proceso de traer a la memoria que hay infinitos números primos (the process of recalling that there are infinitely many primes).

Según Williamson, "ver", "recordar", "pudo oír", "pudo sentir", y "tener una prueba de que" son FMSO. "Conocer" posee indiscutiblemente todas las características de los FMSO excepto una. No es claro que no pueda ser analizado semánticamente. Williamson dedica varios párrafos a argumentar en contra de la empresa de analizar al conocimiento, y considera que los sistemáticos fracasos asociados a ella son prueba suficiente de la inanalizabilidad del conocimiento. No me interesa criticar en este punto a Williamson, de modo que concederé contextualmente que el conocimiento es inanalizable. Si esto es así, entonces "conocer" es un FMSO. La inanalizabilidad semántica de los FMSO a su vez reflejaría la no composicionalidad de las actitudes factivas estativas asociadas. De este modo, si "conocer" es un FMSO, entonces (CEMf) es verdadera: Hay un estado mental (no descomponible en factores internos y externos) necesario y suficiente para conocer.

El próximo paso de Williamson es ofrecer una explicación teórica del conocimiento a partir del reconocimiento de la relación de "conocer" con los otros FMSO. Siempre que decimos que un sujeto tiene un FMSO hacia una proposición, podemos concluir que el sujeto tiene la relación de conocimiento hacia dicha proposición. Esta relación se da únicamente con el FMSO "conocimiento", y se expresa mediante los principios (a) y (b).

(a) "Conocer" es un FMSO.

(b) Si $\Phi$ es un FMSO, entonces uno puede inferir de "S $\Phi$ que p" que "S sabe que p".

Según Williamson, ambos principios "caracterizan al concepto de conocer de modo único, hasta la equivalencia lógica, en términos de un FMSO" (2000, p. 39). Sin embargo, esa expresión es un poco desafortunada. Como él mismo afirma más tarde (2009, pp. 289-290), lo que estos principios caracterizan es más bien al conocimiento mismo, lo 
cual es coherente con la frase que engloba a estas ideas: "El conocimiento es la actitud factiva estativa más general", la cual refiere al conocimiento mismo y no a su concepto.

Con esta explicación del conocimiento, Williamson obtiene una ganancia teórica fundamental, que es la primera razón a favor de (CEMf) que podría sopesar las consideraciones que apuntan al elemento social del conocimiento: explica de un modo simple la fundamental cuestión del valor del conocimiento:

El conocimiento es la actitud factiva estativa más general, aquella que uno tiene hacia una proposición si uno tiene una actitud factiva estativa en absoluto [...] El punto de esta conjetura es iluminar el rol central del concepto de conocer en nuestro pensamiento. Nos importa porque las actitudes factivas estativas nos importan (2000, p. 32). ${ }^{14}$

Los estados mentales factivos nos importan como estados cuya esencia incluye una correspondencia entre la mente y el mundo, y conocer nos importa como la actitud factiva estativa más general. Por supuesto, algo debe decirse sobre la naturaleza y la significación de esta correspondencia, pero ese es un problema posterior (2000, p. 40).

Mientras la creencia apunta al conocimiento, varios procesos mentales apuntan a estados mentales factivos más específicos. La percepción apunta a percibir que algo es el caso; la memoria apunta a recordar que algo es el caso. En la medida en que el conocimiento es el estado factivo más general, todos esos procesos apuntan a tipos de conocimiento. Si una criatura no pudiera engranarse en esos procesos sin alguna capacidad de éxito, podemos conjeturar que nada podrá tener una mente sin tener una capacidad para conocer (2005, p. 2).

En resumidas cuentas: nos importa el éxito de nuestras acciones. Nos importan las actitudes factivas porque nos garantizan este éxito y el conocimiento nos importa porque es la actitud más general.

\subsubsection{Los FMSO bajo la lupa (un filtro lingüístico)}

El rol central de la percepción y la memoria en las consideraciones sobre el valor del conocimiento es ineludible. Mostraré que los términos

${ }^{14}$ Las itálicas son mías. 
correspondientes a ellos en el lenguaje natural no pertenecen a la categoría de los FMSO. Argumentaré que no cumplen con una o más condiciones de las necesarias para ser un FMSO. Utilizaré para esto solo estrategias y categorías pertenecientes a la lingüística. Las categorías fundamentales serán los esquemas temporales propuestos por Vendler (1967), de modo que utilizaré las mismas herramientas teóricas que Williamson.

El rol fundamental de estas categorías es el de relacionar ciertas características temporales de los verbos con los conceptos a los que refieren. En este sentido, si un verbo pertenece a más de una categoría, puede suceder que haya más de un concepto detrás del mismo. Los esquemas de tiempo son actividad, realización, logro y estado. Los primeros dos, a su vez, son procesos que ocurren en el tiempo y los segundos, no.

A grandes rasgos, los términos de actividad tienen tiempos continuos (está trabajando, corriendo, escribiendo, etc.) y piden períodos de tiempo que no son únicos ni definidos. Algunas preguntas como "¿Por cuánto tiempo estuviste escribiendo/corriste/bailaste/etc.? se adecuan a esta categoría (aunque no la implican porque dichas preguntas se adecuan también a términos de estado).

Los términos de realización, si bien también poseen tiempos continuos (está construyendo un edificio, dibujando un robot, escribiendo un artículo, etc.), piden períodos de tiempo definidos, pues poseen una suerte de "punto terminal" que resignifica toda la acción. De este modo, si alguien construye un edificio en dos años, no es cierto que haya construido un edificio en cualquier parte de ese período. Por esta razón, preguntas como “¿Cuánto tiempo te llevó construir el edificio?” son más acordes a esta categoría.

Los términos de logro, por su parte, no admiten tiempos continuos y pueden ser predicados solo por momentos definidos, singulares. La acción entera lleva solo un instante (alcanzar la cima de una montaña, dar en el blanco, etc.). Vendler señala la característica fundamental de estos términos que es que el tiempo presente casi no se usa (excepto como presente histórico o indicando un futuro inmediato). ${ }^{15}$

Los términos de estado requieren alguna consideración adicional. Tampoco admiten tiempos continuos y pueden ser predicados por períodos cortos o largos de tiempo (él cree que las fallas se deben al azar, él sabe cómo comportarse, él posee agallas, etc.). Esta es, sin duda, la categoría más difícil con la que trabajar. Toda propiedad puede verse como un estado

15 Puedo decir "lo he visto" tan pronto como pueda decir "lo veo". 
(ser rojo, ser un escritor, ser alpinista, ser alto, ser amante de las frutillas, etc.). Por esta razón, debemos tener especial cuidado en no atribuir rápidamente al concepto relacionado con un término con apariencia de estado las propiedades de esta categoría. Otra característica, derivada de esto, que los términos de estado poseen, es que pueden predicarse de sujetos sin que estén actualmente involucrados en ningún proceso o logro en particular. En este sentido, puedo predicar de un hombre que duerme que "cree en Dios, que ama los gatos, que es un alpinista, etc". Llamemos a esta característica independencia de predicación.

Tanto las actividades, como las realizaciones y los logros tienen, en su mayoría, un sentido de estado derivado. Hay dos modos en que los estados pueden asociarse a otra categoría. Una actividad, realización o logro específicos pueden producir un estado específico. Alguien "es un fumador" si realiza la actividad de fumar, alguien "es un escritor de libros" si escribe libros (que es una realización), y, por supuesto, alguien es un "bateador" en caso de que batee (que es un logro). Sin embargo, hay otro tipo de estados que no pueden atribuirse a ninguna categoría específica, sino que se aplican correctamente a un sujeto cuando realiza una serie de actividades o logros diferentes. Por ejemplo, el estado de "ser un líder" no está asociado a una actividad específica de "liderar". No existe algo así como "en este preciso instante estoy liderando". Más bien el estado de "liderar" se da cuando se cumple con una serie elementos más o menos determinables como tener un cargo, llevar una agenda, ser respetado, etc. Estos estados son los estados genéricos, y, según Williamson, el conocimiento sería uno de ellos. No es claro a qué categoría de las dos pertenecerían los términos de percepción y de memoria.

Hay una última consideración a tener en cuenta. Vendler reflexiona sobre la relación estrecha que se da entre una característica sintáctica y una propiedad semántica que no está ligada a cuestiones de tiempo. Aquellos logros que no son voluntarios y todos los estados (que, aunque provengan de un logro o actividad voluntaria no diríamos que son, qua estados, voluntarios), comparten la característica de que, si el término es X, este es frecuentemente intercambiable con "poder X". De este modo, en condicionales como "si ciertas condiciones se dieran, yo podría ser líder" es lo mismo que decir "si ciertas condiciones se dieran, yo sería líder". Igualmente, "puedo reconocerte" es lo mismo que decir "te reconozco", en tanto que "reconocer" es un logro no voluntario. "Podría empujarte" o "puedo empujarte" no es intercambiable con "te empujaría" o "te empujo", en tanto que "empujar" es un logro voluntario.

¿Qué hay, entonces de los términos que Williamson considera FMSO? A partir del breve excurso sobre los esquemas de tiempo, estamos 
en condiciones de evaluar qué es lo que Williamson tiene en mente cuando afirma que los FMSO son estativos y si los términos de percepción realmente caen bajo esa categoría.

Williamson comete un error grave en relación con la mayoría de los supuestos candidatos a FMSO. Si prestamos atención a estos, veremos que muchos están conjugados en pretérito perfecto, como "vio", "pudo ver", "pudo oír", "pudo sentir", etc. ¿Pueden ser usos de estados? Como es obvio, no puedo decir de un hombre dormido que "vio que estaban cortando un árbol" con la intención de predicar un estado (un estado que sería algo así como "ser un vidente en el pasado de la cortadura de un árbol"), no del mismo modo en el que decimos que "sabe que dos más dos es igual a cuatro". La lectura obvia de la primera oración es que más bien estoy relatando un logro, algo que tuvo lugar en un instante determinado de tiempo. Como es aún más transparente, "pudo ver" y "pudo oír" refieren claramente a logros y no a estados. ${ }^{16}$

Esto parece expulsar de un tirón a un conjunto central de términos del de los FMSO. Sin embargo, esto no descarta la posibilidad de que el sentido derivado de estado de esos términos sea un FMSO, es decir, que sean estados específicos asociados a los términos de logro relacionados. De este modo, si bien "pudo ver" no es un FMSO, a lo mejor "puede ver" sí lo es, y otro tanto podría suceder con los estados derivados de "ve", "puede oír" y "puede sentir". Evaluemos entonces si dichos términos son FMSO.

Comencemos por "ve". Como hemos visto, "ver" posee un sentido claramente perceptivo. Pero este sentido no puede preservarse como estado, pues "ve que el hombre avanzó" no preserva su sentido perceptivo a menos que se piense que el sujeto está actualmente viendo que el hombre avanzaba, es decir, no parece tener la característica que hemos llamado independencia de predicación y que es propia de los estados. "Ve en el diario que la bolsa subió" es igual de impropio por las mismas razones ${ }^{17}$. "Ver", sin embargo, parece tener un segundo sentido además del perceptivo, que se manifiesta en oraciones como:

16 Cuando anteceden a la cláusula "que" (es decir, en la adscripción de una actitud proposicional) cumplen con todas las características de los logros: (1) puedo decir "pude ver"/"pude oír" en el instante en que diga "puedo ver"/"puedo oír"; (2) ocurren en un instante determinado de tiempo; y (3) el presente "puede ver"/"puede oír" es poco frecuente.

${ }^{17}$ La posibilidad del uso del presente, como en "ve que dos hombres se le acercan" y "oigo que está martillando", no es índice de estado. Estos usos están perfectamente habilitados en tanto actividades, como es en "en este momento Juan corre la maratón". Como ya he argumentado, sería absurdo predicarlos de un hombre que no esté, a la vez, involucrado en la actividad correspondiente de ver, oír o correr. 
(15) Y entonces pude ver con claridad que habíamos sido dos adolescentes ingenuos.

(16) Veo que tenés un punto, vamos a tomarnos unos minutos para pensar antes de seguir con la discusión.

(17) Mi hermana puede ver que el ejercicio está mal, ese no es el problema, el problema es que no entiende por qué.

Este sentido es el que está presente en oraciones como (3). Permite pasar de "Vio que tenía razón" a "Ve que tenía razón" sin problemas. Pero rápidamente notamos que este sentido de ver es muy diferente del perceptivo, el cual exige que (entre otras capacidades) la capacidad visual esté involucrada. Esta es la razón por la cual un sujeto no vidente no puede "ver que una mujer se acerca" ni "ver en el diario/noticiero que la bolsa subió dos puntos". Sin embargo, un no vidente puede "ver que fue engañado", "ver que se equivocó", y "ver que había razones para aceptar la evidencia desencaminada". Pero este sentido de ver es completamente reemplazable por "entiende" o "comprende". No tiene nada que ver con la visión excepto algunas características "fenomenológicas" que podrían explicar que haya un sentido de "ver" asociado a la comprensión. Me refiero a que cuando vemos, es decir, cuando nuestro aparato visual se pone en funcionamiento, tenemos la sensación de "apertura" hacia el mundo, de que las cosas están ahí sin intermediación alguna. Algunas veces, cuando pensamos claramente un problema y llegamos a entenderlo de raíz, sentimos que podemos asirlo, que realmente lo vemos. Pero no debemos dejar que este sentido nos confunda, pues es un sentido metafórico parafraseable o, mejor aún, reemplazable por "entiende claramente que", "comprende" o "entiende" a secas.

Habría entonces dos sentidos de "ver":

-"Ver" en el sentido perceptivo. Puede ser un término de logro, o bien uno de actividad. ${ }^{18}$ Como en "Carlos vio que la mujer bailaba" y "todo el mundo está viendo que te sonrojas".

-"Ver" en el sentido de comprender, puede ser un término de logro, o bien uno de estado genérico. ${ }^{19}$ Como en "el dueño de la plantación vio que los osos pandas merecen vivir mejor" y "aunque no se lo digas, Alberto ve claramente que vas a marcharte, no es idiota".

18 Una advertencia sobre los nombres. La categoría de actividad incluye procesos espontáneos como los de respirar.

${ }^{19} \mathrm{El}$ hecho de que Vendler no haya distinguido estos sentidos de modo claro es lo que explica su largo argumento a favor de que "ver" es un estado genérico, en contra de la tesis de que es una actividad. Los argumentos son francamente forzados (dependen 
Volvamos ahora a nuestra pregunta sobre si "ve" puede ser un FMSO. Según lo que he dicho, "ve" tiene una lectura que podría ser estativa (la lectura asociada a la comprensión) y otra de logro o actividad (la lectura asociada la percepción). Williamson descarta como evidencia a favor de que "recordar" es una actividad el hecho de que la lectura más saliente de "recordando" es "está trayendo a la memoria". ¿Podríamos hacer lo mismo con las lecturas de logro y actividad de "ver"? Creo que no solo no podríamos (¿sobre qué clase de argumentos negaríamos que el sentido básico de "ver" es perceptivo?) sino que deberíamos hacer lo inverso, descartar la evidencia a favor de que "ve" es un término de estado admitiendo que la lectura más saliente de "ve" cuando se predica, por ejemplo, de hombres dormidos (es decir cuando está presente la independencia de predicación), es la de "comprende". "Entender" o "comprender" es un estado y es factivo. Si decimos, a la vez, que no es analizable semánticamente, debemos aceptar que es un FMSO, lo mismo que "puede entender", debido a la intercambiabilidad característica de estados y logros no voluntarios. Pero el sentido saliente de "ver" no es ése. El sentido saliente de ver no es estativo y, por lo tanto, "ver" no es un FMSO. A la vez, si se acepta hasta aquí mi argumentación, "puede ver no es distinto de "ve", pues que no sería otra cosa que el término intercambiable con "ve" propio de los logros no voluntarios. ${ }^{20}$ "Puede ver" equivale a "ve", como en "María puede ver que el cuadro es rojo", "Puedo ver que me sigues" etc, y por lo tanto tampoco es un FMSO.

Lo que intento mostrar aquí no es que "ver" y las expresiones asociadas tengan un único significado y que este no sea el estativo mencionado por Williamson. Más bien, busco mostrar algo más débil, a saber, que si hay un uso estativo de "ver" (ya sea por polisemia o por ambigüedad), ese uso no es el perceptivo sino el asociado a la comprensión o entendimiento.

Podemos extraer las mismas conclusiones para "puede oír" y "puede sentir" que extrajimos para el sentido saliente de "ver". "Puede oír" no posee ningún sentido de estado, y "puede sentir" (que rescata el sentido perceptivo de logro menos usual de "siente" y posee un sentido usual de "percibe con el sentido del tacto") se corresponde con un logro (perceptivo).

de consideraciones tan débiles como que ver no es una actividad porque sería extraño responder a una pregunta como "¿Qué estás haciendo?" algo como "Estoy viendo..." -extrañeza perfectamente explicable mediante la apelación a las máximas conversacionales griceanas- y omisiones graves como el hecho de que ver es, como respirar, un proceso espontáneo).

${ }^{20}$ No necesito defender aquí que la lectura saliente de "puede ver" es la perceptiva. Hasta Williamson lo admite para "pudo ver". 
Ninguno de ellos es un FMSO, pues ninguno de ellos es, propiamente, un término de estado.

Para concluir esta subsección, quiero ofrecer un argumento adicional en contra de que los términos perceptivos son FMSO. Este argumento vale también para "recordar". Aunque el foco de este trabajo está en los términos de percepción, quiero al menos motivar la idea de que "recordar" tampoco es un FMSO.

"Conocer" es, sin duda, el caso paradigmático de los términos factivos. La evidencia lingüística a favor de ello es muy fuerte. No se basa solo en la incorrección de expresiones como "Juan sabe que la tierra es cuadrada, no tiene idea de lo equivocado que está" o "Saber algo falso es lo peor que a uno le puede pasar". Esas "infelicidades" están acompañadas de otros hechos lingüísticos como la imposibilidad de usar ciertos modificadores para "conocer". En palabras de Vendler “...una afirmación como uno no puede saber algo incorrectamente es verdadera en todos los lenguajes siempre y cuando sea traducida adecuadamente" (1967, p. 26). Creo que hay una diferencia muy grande entre los términos de percepción y "conocer" en ese punto. Tenemos un conjunto de modificadores, como "incorrectamente", "correctamente", "erróneamente", "falsamente", que se aplican a los primeros y no al segundo. En inglés existe incluso el prefijo "mis" que se aplica usualmente al fenómeno de la percepción (misperception), de modo que podemos ver mal, ver erróneamente, ver correcta o incorrectamente, etc., mientras que dicho prefijo es inadecuado para "conocer". ${ }^{21}$ Otro tanto sucede con los casos de memoria "no recuerdo muy bien qué pasó", "recuerda erróneamente que", etc. ${ }^{22}$

El caso a favor de la pretendida factividad de los términos de percepción se reduce a un único tipo de evidencia: la aparente impropiedad de cosas como "oyó que la puerta se cerró pero en verdad estaba abierta", "vio que había palabras en la pizarra pero no había nada realmente", y "recordó que sus llaves estaban en el escritorio para luego darse cuenta de que las tenía en el bolsillo".

${ }^{21}$ La aplicación de esos modificadores es igualmente incorrecta para los términos "pudo ver", "pudo sentir" y "pudo oír" considerados por Williamson, de modo que estas reflexiones no se aplican a los mismos. Sin embargo, estos términos son los que, desde el punto de vista de las categorías temporales son más claramente no estativos (por los argumentos esgrimidos arriba). El propósito de estos comentarios finales es mostrar que no solo hay razones lingüísticas para pensar que términos como "ver" y "recordar" no son estativos (y por lo tanto no son FMSO) sino también para pensar que no son factivos. Agradezco a un árbitro anónimo por hacerme notar este punto.

22 El habla en términos de "recuerdos falsos" es ubicua. 
Por supuesto, usualmente cuando decimos que alguien escuchó que la puerta se cerraba, implicamos que la puerta se cerró; cuando decimos que alguien vio que la pizarra estaba escrita, implicamos que la pizarra estaba escrita; cuando decimos que alguien recordó que sus llaves estaban arriba del escritorio, implicamos que sus llaves estaban arriba del escritorio, y así. Pero también es cierto que estamos habilitados a decir cosas como "Houston, tenemos un problema. El astronauta Javier ve que el satélite gira para la izquierda, mientras que el astronauta Germán ve que gira para la derecha. En este caso Javier está en lo correcto, pero sistemáticamente ven que las cosas giran hacia lados opuestos", "Juan recuerda que su padre era valiente, pero, en realidad, era un hombre muy cobarde", "Ana está consternada, porque ve que un sujeto se le acerca, pero sabe que es una sombra", "Alberto oye que está sonando una melodía, pero en realidad es una pava bullendo".

La diferencia entre "conocer" y los términos de percepción en estos puntos es, además, perfectamente explicable apelando a la noción de presuposición. Ya hemos visto que Williamson la utiliza para términos como "adivinó". Los términos de percepción, bajo esta interpretación, presupondrían la verdad de la proposición, pero esta presuposición sería cancelable por contexto. ${ }^{23}$

\subsubsection{Los FMSO y el valor del conocimiento}

¿Cómo repercuten estas consideraciones en la tesis de que el conocimiento es la actitud factiva estativa más general? Si los términos de percepción -y, tal vez, los de memoria- no son FMSO, la caracterización del conocimiento en términos de la categoría de los FMSO mediante los principios (a) y (b) deja de ser atractiva desde el punto de vista teórico. Por un lado, ya no hay razones basadas en el estudio del

${ }^{23}$ Hace algunos años la factividad de los términos de percepción se ha tornado una aparente trivialidad. Pero esto no ha sido siempre así. Filósofos como Chisholm (1957, 1976) y Ayer (1956) consideraban trivial la tesis de que no lo eran. Ambos extremos, creo, corresponden con tesis filosóficas robustas que "legislan" hacia el plano del lenguaje en casos en los que la evidencia lingüística no es concluyente. Así el "problema de la percepción", tal como se discutía hace cincuenta años, favorecía la tesis de que nuestro uso de los términos de percepción permitía la posibilidad de percibir que algo era el caso cuando en verdad no lo era. En el presente, parece haber consenso sobre la idea de que "ver que algo es el caso" implica que hay algo que es el caso. Pero detrás de este "descubrimiento" lingüístico están, por ejemplo, las reflexiones de Dretske (1969) y Putnam (1985), quienes rechazan la idea de que haya un fenómeno natural al que propiamente corresponda el de la percepción (en el caso de Putnam) o bien que si lo hay, 
lenguaje para pensar que los estados perceptivos -y, tal vez, de memoriason actitudes factivas estativas. De modo más general, la categoría misma de FMSO parece más un constructo ad hoc que una genuina categoría lingüística. Por otro lado, la caracterización del conocimiento como actitud factiva estativa más general ya no explica el valor del conocimiento, en tanto que este se explicaba en virtud del valor de la percepción y de la memoria. Se pierde así una de las dos razones teóricas fundamentales para considerar admisible la tesis (CEMf).

\subsection{Los argumentos filosóficos}

En lo que resta de esta sección presentaré consideraciones filosóficamente sustanciales en apoyo de las conclusiones basadas en la evidencia lingüística. Incluso si dicha evidencia no fuera confiable, tenemos razones independientes para pensar que aun si hubiera estados perceptivos y de memoria, no serían factivos. Al mismo tiempo, estas reflexiones tendrían legitimidad aun si los términos de percepción fuesen, en efecto, FMSO.

Recordemos que la tesis (CEMf) implica que el conocimiento es una clase natural. Otro tanto sucede con los estados perceptivos y de memoria $q u a$ actitudes estativas factivas. Esto significa que la ciencia, por un lado, y la filosofía de la percepción y la memoria (sea cual sea el lugar que le quedase a esta), por el otro, hablarían de un mismo fenómeno. ${ }^{24}$ Por esta razón, es recomendable tener en consideración lo que la ciencia puede decir sobre la cuestión de la factividad de los procesos perceptivos y de memoria, que son los que darían origen a los estados mentales correspondientes.

La evidencia científica a favor de la falibilidad de los procesos perceptivos y de memoria es abundante. En general, todos los experimentos de alucinación e ilusión se describen de forma tal que los

no tiene injerencia en las reflexiones filosóficas sobre el mismo (en el caso de Dretske). El fenómeno del "ver epistémico", que es el que interesa a Dretske, refiere a un supuesto concepto que resulta en un análisis de "ver que" muy similar al de "conoce que" (excepto porque refiere a la visión). El término correlativo a este concepto es, por supuesto, factivo, pero no debemos confundir una tesis lingüística que posee apoyo fundamentalmente de una tesis filosófica sustantiva como es la de Dretske, y que por lo tanto posee una forma como "este término debe comportarse de cierto modo" con una tesis lingüística supuestamente trivial de una forma como "este término se comporta así".

24 Esto es lo que hace impertinente utilizar los argumentos de los autores mencionados en la nota anterior a favor de la factividad de "ver" para esta propuesta en particular. Ninguno de ellos pretende estar hablando de tipos naturales. 
sujetos efectivamente perciben que (hay una rata rosa, hay algo que los golpea, etc.). ${ }^{25} \mathrm{~A}$ su vez, hay fuertes indicios de que el lugar de la imaginación en la memoria es central, lo cual está estrechamente conectado con la posibilidad de recuerdos falsos. ${ }^{26}$ Esto es completamente coherente con la idea de que los términos perceptivos admiten modificadores como "correctamente", "incorrectamente", "erróneamente", etc.

Por otro lado, hay una línea argumental de tinte evolutivo basada en estudios empíricos y los hallazgos científicos asociados. Consiste en la tesis de que los estados perceptuales podrían proveer representaciones inadecuadas no solo porque fallan en ciertas ocasiones - este sería el caso de las alucinaciones o las ilusiones-, sino porque el objetivo de la percepción no sería el de proveer representaciones adecuadas. ${ }^{27-28}$ Los hallazgos son importantes, de acuerdo con Viger:

La discriminación de colores requiere al menos dos tipos de fotorreceptores con sensitividades espectrales diferentes. En nuestro caso, tenemos tres de esos fotorreceptores, o conos, que responden preferencialmente a rangos diferentes de la banda visible de radiación electromagnética (entre 400 y $700 \mathrm{~nm}$ ). Algunos conos responden mejor a la luz azul, otros a la verde, y otros a la roja. Las respuestas son preferenciales pero no exclusivas a espectros particulares de luz porque la activación de un cono es causada por una interacción de quantum entre los fotones y el pigmento en el cono. Hay solo una posibilidad estadística de que la interacción ocurra, de modo que los conos no siempre responden incluso a la luz a la cual son más sensibles, y cualquier tipo de cono puede responder a cualquier espectro de luz. Los colores son determinados por el cerebro basándose en las respuestas diferenciales entre los grupos de tipos de conos. [...] el sistema está organizado para maximizar el contraste entre los estímulos verde/rojo y azul/amarillo [...]. La consecuencia para nuestra discusión es que fenomenológicamente vemos los mayores contrastes entre rojo/verde y azul/amarillo, consistentemente con, y a causa de, nuestra organización fisiológica. Esta, sin embargo, no es la verdadera estructura de la

25 Tan solo para mencionar algunos: Gregory (1968), Morrison y Wells (2000), Laroi y Van der Linden (2005), Kitaoka, Gyoba y Sakurai (2006), Girgus, Coren y Fraenkel (1975).

${ }^{26}$ Waring, Payne, Schacter y Kensinger (2010), Szpunar y Schacter (2013).

27 Esto no contradice, sin embargo, la idea de que mayormente percibimos correctamente. Por lo general, lo útil es lo verdadero.

${ }^{28}$ Se ha seguido esta misma línea para el caso de la memoria en Schacter, Guerin y St. Jacques (2011). 
radiación electromagnética que visualmente detectamos. Los espectros de la luz que vemos como roja son mucho más cercanos a aquellos de la verde que a la del azul, aunque el rojo y el azul nos parezcan más similares a nosotros (Viger 2006, pp. 276-277).

Esto se debería a cuestiones adaptativas que facilitarían la supervivencia. Viger $(2006)^{29}$, refiere a varios experimentos, uno para el sentido del tacto, otro para la visión, y otro para la audición. Luego de presentar el caso experimental para la percepción visual ${ }^{30}$, observa:

No solo nuestra percepción de colores falla en proveer representaciones adecuadas, puede ser adaptativo que así sea. [...] la percepción del color es como el "falso color" utilizado en la telemetría satelital. Características físicas arbitrarias que no tienen nada en común excepto el hecho de que afectan a alguno de los detectores del satélite del mismo modo son representados como teniendo el mismo color. Desde el punto de vista de la posición de ventaja del satélite, no percibiríamos la escena tal como es representada, pero la representación hace que las diferencias reales sean más "vistosas" (Viger 2006, p. 277).

El contraste útil es la llave para el éxito perceptivo y no la fidelidad de las representaciones (Munevar, citado por Viger 2006, p. 277).

Esta línea argumental puede no ser concluyente, pero cuando menos debemos admitir que aquello en lo que se originan estas consideraciones es en desarrollos científicos que fijan los "límites" de la percepción de un modo tal que podemos "efectivamente percibir" que algo es el caso cuando no lo es.

Estas ideas sobre la percepción y la memoria tienden a mostrar que, aun si la evidencia lingüística fuese inconcluyente, la siguiente afirmación condicional es verdadera: si hubiera estados perceptivos y de memoria, estos no serían factivos (y por lo tanto no serían actitudes factivas estativas). De este modo, se fortalece el argumento en contra de que la tesis del conocimiento como actitud factiva estativa más general explique adecuadamente el valor del conocimiento.

29 Él sigue a Clark (2002) en su defensa de la tesis de que el propósito de la percepción no sería el de proveer representaciones adecuadas. Otro gran defensor de esta tesis es Hoffman (2011). Véase también Hoffman, Mark y Marion (2010).

${ }^{30}$ Véase Kandel, Schwartz y Jessell (1995, pp. 453-468) 


\section{La explicación de la acción}

Como dije anteriormente, una de las virtudes que esgrime Williamson a favor de (CEMf) es la de satisfacer la necesidad de postular un estado mental de conocer para explicar causalmente una serie de acciones que de otro modo no podrían explicarse adecuadamente. En esta sección muestro que no hay tal necesidad teórica puesto que contamos con la creencia racional para dar cuenta de dichas acciones.

El foco de la explicación causal de la acción está puesto, más que en la intención de llevar a cabo la acción, en aquello requerido para que la acción haya sido efectivamente llevada a cabo. (Williamson 2000, pp. 60-64) La idea es que los estados mentales legítimos ocupan un lugar en la explicación causal de alguna acción.

$\mathrm{El}$ argumento de Williamson depende fundamentalmente de la idea de que los estados mentales legítimos poseen eficacia causal. Aunque la noción de eficacia causal no es absolutamente clara, Williamson sugiere que, sea cual sea esa noción, es bastante plausible pensar que los estados mentales que poseen eficacia causal deben ser indispensables para la explicación adecuada de algún tipo de acciones (2000, p. 61).

La noción de "explicación adecuada" está asociada a la idea de que el explanandum (la acción) y su explanans (el estado) han de tener una correlación alta, donde esto significa que la probabilidad condicional del explanandum dado el explanans ha de ser alta, y que la probabilidad condicional del explanandum dada la ausencia del explanans es baja. Lo primero refleja el poder predictivo de las buenas explicaciones, y lo segundo el carácter general de las mismas (2000, p. 83). Pero una alta correlación no es un indicador infalible de relación causal (2000, p. 88), ni el único indicador a considerar a los fines de obtener la mejor explicación:

[...] la baja de la probabilidad condicional del explanandum dado el explanans [no] implica estrictamente una pérdida de poder explicativo; solo redunda en dicha pérdida cuando [...] no hay ganancias compensatorias (2000, p. 62).

De este modo, la noción misma de "explicación adecuada" también incluye otras virtudes explicativas como la simplicidad y la consistencia teórica.

Williamson, de manera no sorprendente, señala que el conocimiento ocupa un lugar central en la explicación de ciertas acciones y que no puede sustituirse por ninguna combinación de creencia, verdad, justificación, etc., sin una pérdida sustantiva de poder explicativo. 
¿Por qué se seguiría (CEMf) de esto último? Consideremos la primera explicación que toma en cuenta Williamson: "Él desenterró el tesoro porque sabía que estaba enterrado bajo el árbol y quería hacerse rico" (2000, p. 61). El internista podría argumentar que el conocimiento no ocupa el lugar pretendido en esa explicación pues "sabía" puede sustituirse sin pérdida explicativa por "creía con verdad". Creer con verdad es un estado que posee el mismo nivel de correlación con ese explanandum que conocer, y por ende puede sustituirlo sin pérdida explicativa. Por supuesto, creer con verdad no es un estado mental genuino. Es una condición descomponible en un estado interno al sujeto (la creencia) y un estado externo (el hecho en cuestión).

Si hay acciones para las que esta posibilidad de sustitución sin pérdida de poder explicativo no es posible, entonces la mejor explicación de ese hecho es que el conocimiento sea un estado mental genuino (no descomponible).

El tipo de acciones a las que apela Williamson para mostrar esto último son aquellas en las que el éxito de la acción está relacionado con la resistencia a la contraevidencia por parte del sujeto. La idea es que la actitud positiva hacia p es más robusta y estable en el caso de conocer que en el caso de una mera creencia verdadera. Ciertas acciones que se llevan a cabo a lo largo de un período de tiempo extendido (por ejemplo, registrar una casa ajena durante toda la noche en busca de un diamante) no tienen la misma probabilidad si meramente se cree que el diamante está en la casa que si esto se sabe. Eso es así porque la mera creencia (aunque casualmente verdadera) puede ser sostenida por razones falsas, o débiles, de modo que cualquier contraevidencia, por escasa que sea, podría hacer que el sujeto dejase de sostenerla. El mero paso del tiempo sin éxito podría debilitar la creencia de que el diamante está en la casa. El aporte distintivo del conocimiento a la explicación causal de ciertas acciones sería, entonces, la robustez frente a nueva contraevidencia en casos de acciones prolongadas en el tiempo.

Williamson tiene en cuenta otros posibles sustitutos como "creencia verdadera basada solo en razones verdaderas", "certeza subjetiva" o incluso "creencia irracionalmente insensible a la contraevidencia”. Rechaza la primera opción porque

[...] pueden construirse variantes del caso anterior en las que el ladrón entra a la casa creyendo verdaderamente que hay un diamante, sin basarse en razones falsas, pero careciendo de conocimiento en virtud de la existencia de contraevidencia que, si bien él no posee, podría descubrir en el curso de su búsqueda, y esto haría que abandone el asunto (2000, p. 63). 
Las segundas, en cambio, son rechazadas porque

[...] aunque el conocimiento no es invulnerable a la destrucción por evidencia posterior, su naturaleza es ser robusto en ese respecto [...] [las otras opciones representan] un tipo de tozudez diferente; no pueden reemplazar al conocimiento en todos los contextos de explicación causal, por la simple razón de que aquellos que saben que $\mathrm{p}$ frecuentemente carecen de una creencia necia sobre p. No es necesario que la creencia del ladrón sea necia. Similarmente, no es necesario que sienta certeza sobre ella; la certeza subjetiva no puede siempre reemplazar al conocimiento (2000, p. 63).

A partir de este último ejemplo, podemos especificar un poco más el aporte distintivo del conocimiento: robustez frente a nueva contraevidencia, pero sensibilidad a la misma.

En contra de Williamson, considero que la creencia racional realiza el mismo aporte que el conocimiento y, por lo tanto, funciona como un eficaz sustituto. El ladrón creía racionalmente que el diamante estaba en la casa, de modo que pasó toda la noche revisando la casa. Es extraño que Williamson no considere esta opción. Más allá de la concepción específica que se tenga de racionalidad, si algún concepto está asociado a la sensibilidad a razones o evidencia, este es el de racionalidad. Una creencia no puede ser racional si no es sensible a la evidencia, donde esto significa que la creencia es adecuada en función de la evidencia disponible al sujeto. Podemos esperar, entonces, que la creencia no se debilite frente a la presencia de cualquier tipo de contraevidencia nueva, pero sí lo haga si la contraevidencia que surge es abrumadora. Y esto es precisamente lo requerido: un balance entre firmeza y sensibilidad ante nueva contraevidencia. Esta concepción de creencia racional obviamente no excluye la posibilidad de que haya una creencia racional falsa. De hecho, para funcionar como sustituto de conocimiento no es necesario asumir que la creencia racional sea además verdadera, sino meramente que el fenómeno de la creencia racional no está desconectado de la verdad, es decir, que la probabilidad de que una creencia de que p sea verdadera, dado que es racional, sea mayor a la probabilidad no condicional de que p. ${ }^{31}$

${ }^{31}$ Esto significa que si una persona cree racionalmente que $\mathrm{p}$, entonces $\mathrm{p}$ es probable objetivamente. Aunque esta noción de racionalidad es bastante común, deseo considerar alternativamente una opción para quienes defienden una concepción de la racionalidad según la cual una creencia es racional cuando su probabilidad subjetiva es alta. Aunque dudo que incluso esta noción de racionalidad esté desconectada de la verdad (excepto 
Deseo aclarar, en este sentido, que no es mi intención mostrar que la creencia racional pueda sustituir al conocimiento con el mismo nivel de correlación con las acciones exitosas (pues el conocimiento implica la verdad de la proposición y la creencia racional solo la hace probable) pero sí mostrar que la correlación es lo suficientemente alta como para no perder poder explicativo. Recordando a Williamson, "la baja de la probabilidad condicional del explanandum dado el explanans no implica estrictamente una pérdida de poder explicativo; solo redunda en dicha pérdida cuando [...] no hay ganancias compensatorias" (2000, p. 62). Si se sustituye al conocimiento como estado mental genuino por la creencia racional (i.e., sensible a la evidencia), la ganancia compensatoria es muy clara: evitamos postular un estado mental sui géneris distinto del de creencia.

¿Qué podría responder Williamson a esta propuesta? No creo que los argumentos para otros sustitutos pudieran ayudarlo. No podría argumentarse, por ejemplo, que la creencia racional no es suficientemente robusta porque es sensible a demasiada evidencia en contra. Una eventual retirada frente a contraevidencia abrumadora es lo que se necesita, según Williamson, para distinguir la robustez propia del conocimiento de la terquedad propia de una creencia necia. Aun un sujeto que sabe puede perder la confianza (y dejar de revisar la casa) frente a una cantidad suficiente de contraevidencia. ${ }^{32}$

Si no hay acciones para las que el conocimiento no pueda reemplazarse por creencia racional, entonces no hay razones para pensar que el conocimiento es un estado mental genuino. Con esto queda en claro, contra Williamson, que ser la única teoría capaz de explicar ciertas acciones no es una virtud teórica de (CEMf). ${ }^{33}$

para quienes consideran seriamente escenarios de cerebros en cubetas, los sujetos racionales suelen tener creencias verdaderas), es cierto que la relación entre la racionalidad de $\mathrm{p}$ y la probabilidad objetiva alta de $\mathrm{p}$ no es tan directa. Pero esta noción más internista de racionalidad puede complementarse con la noción de verdad. De este modo, quienes defienden dicha noción pueden sustituir conocimiento por "creencia racional $y$ verdadera" en los casos considerados por Williamson, manteniendo de este modo la correlación alta entre explanans y explanandum.

32 Sería un caso desafortunado - pues si el sujeto sabía, entonces la "abrumadora" contraevidencia será engañosa- pero posible. Williamson afirma explícitamente que el conocimiento es, aunque robusto, derrotable frente a contraevidencia abrumadora: "aunque saber no es invulnerable a la destrucción por evidencia posterior, su naturaleza es ser robusto en ese sentido" (2000, p. 63).

${ }^{33}$ En este sentido, la creencia racional sería un genuino estado mental. Un genuino estado mental no factivo. 


\section{Una posible respuesta de Williamson}

Hay un argumento que Williamson podría esgrimir en respuesta a mis objeciones, basado en una defensa que él realiza de la necesidad de postular actitudes factivas estativas perceptivas en general. ${ }^{34}$ La idea central es que es necesario postular dichas actitudes para dar cuenta de ciertas acciones temporales. Si esa defensa es válida, entonces Williamson podría restituir la plausibilidad de la tesis de que el conocimiento es la actitud factiva estativa más general a partir del siguiente argumento:

1) Deberíamos postular las actitudes factivas estativas correspondientes a los términos de percepción y memoria si queremos brindar explicaciones causales exitosas de algunas acciones.

Lo cual ofrece un camino más o menos directo para argumentar:

2) Después de todo, la tesis de que el conocimiento es la actitud factiva estativa más general es verdadera porque hay actitudes factivas estativas de percepción y de memoria - por 1) -, porque es verdadero que si alguien se halla en alguna de esas actitudes respecto de una proposición, entonces conoce esa proposición y porque ambas ideas ofrecen buenas razones para pensar que el conocimiento es un estado mental genuino (aun si este no es indispensable para la explicación de ninguna acción).

Este último punto restituiría, a su vez, la primera virtud teórica de (CEMf) asociada al valor del conocimiento.

La defensa realizada por Williamson a favor de la necesidad de postular actitudes estativas factivas en general sirve para mostrar la verdad de 1), pues los ejemplos que ofrece a favor de la tesis general involucran todos estados perceptivos.

Williamson pone en consideración acciones extendidas en el tiempo que implican una interacción con el entorno y que requerirían para su consecución de estados perceptivos factivos. El fracaso de sustituirlos por otras condiciones manteniendo el poder explicativo es índice (como en el argumento anterior) de que son estados mentales genuinos. El argumento usa el caso de la visión pero puede extenderse a los otros estados perceptivos. Consideremos "bebió el agua" en un caso en el que un sujeto, supongamos que en el desierto, divisa a lo lejos un charco de

${ }^{34}$ La defensa en Williamson (2000, pp. 65-91) apela a varias nociones técnicas y se presenta como un argumento general en favor del valor explicativo de las condiciones "genuinas" (prime) que mostraría la falsedad del internismo de actitudes (2000, pp. 6591). Presento aquí una versión de dicho argumento que recoge únicamente aquellos elementos relevantes para la argumentación de este trabajo, pero la síntesis que realizo no es infiel al espíritu williamsoniano. 
agua y se encamina hacia él (2000, p. 75). La postulación de "vio que había agua" ofrece la mejor explicación para el hecho de que el sujeto llegue exitosamente a beber el agua, porque las probabilidades condicionales de que el sujeto en el desierto beba el agua dado que "cree que ve que hay agua" o "cree que hay agua", o cualquier otro sustituto compatible con la falsedad de que haya agua son mucho más bajos que si el explanans es que "ve que hay agua". En los otros casos podría estar viendo un reflejo del sol similar al agua, un espejismo, o sencillamente estar delirando. Si, en cambio, postulamos que el sujeto "ve que hay agua", entonces, dado que, según Williamson, ver es un estado factivo, la probabilidad condicional de que beba el agua dado que vio que había agua es mucho más alta, pues explica a su vez que haya podido ir registrando, a medida que avanzaba, las distintas direcciones que tenía que ir tomando dada la nueva posición relativa del agua. Todo esto no habría sido posible en caso de que no hubiera agua.

\subsection{No necesitamos postular actitudes factivas}

Estoy de acuerdo con que ningún sustituto puede reemplazar a "vio que había agua", pero considero, primero, que es posible preservar el poder explicativo del explanans sin postular la existencia de un estado factivo $\mathrm{y}$, segundo, que la postulación de estados mentales perceptivos no factivos implicaría una ganancia explicativa en términos de consistencia teórica dadas las consideraciones de las secciones anteriores.

Todo lo que he argumentado anteriormente apoya la idea de que, si vemos que algo es el caso, muy probablemente algo sea el caso o, en general, de que si percibimos que algo es el caso, muy probablemente algo sea el caso. Esto es así en el plano lingüístico, porque la presuposición nos pide que la cancelemos de modo implícito o explícito. También lo es en el plano filosófico porque es parte de la idea de facultad el que sean generalmente confiables -aunque también de que puedan fallar-y en el plano científico porque es condición misma de las conclusiones de los experimentos en que la percepción falla el que esos errores no puedan ser generalizados. De este modo, podemos explicar perfectamente la acción de haber bebido el agua postulando estados de percepción que, aunque no implican la verdad de lo percibido, sí exigen una relación con ella tal que hace muy probable que lo percibido sea verdadero. ${ }^{35}$ Así, podemos ver

35 Si los estados no descomponibles (noción paralela a la inanalizabilidad semántica) pueden ser factivos, también pueden estar relacionados nómicamente con la verdad de un modo no determinista. 
que la probabilidad condicional de beber el agua dado que "vio que había agua" es casi igual de alta cuando ese estado es factivo que cuando no lo es. A la vez, postular actitudes estativas no factivas brindaría mejores explicaciones en virtud de que estaría en armonía con los hallazgos lingüísticos y las consideraciones filosóficas que se derivan de suponer que hay estados mentales de percepción.

Bloqueando de este modo posibles argumentos a favor de 1) la posibilidad de restituir (CEMf) y la tesis de que el conocimiento es la actitud factiva estativa más general queda asimismo bloqueada.

\section{Conclusiones}

En su influyente libro Knowledge and its Limits Williamson ofrece argumentos lingüístico-filosóficos a favor de la tesis (CEMf). Estos dependen de consideraciones preteóricas a favor de la tesis de que el conocimiento es un genuino estado mental y, a su vez, de consideraciones teóricas vinculadas a la caracterización sustantiva del conocimiento como la actitud factiva estativa más general.

En este artículo he mostrado que la tesis (CEMf) es implausible a partir de una objeción novedosa que pretende operar en los dos niveles (preteórico y teórico) considerados por Williamson. De esta forma, la objeción procede en dos momentos. En primer lugar se observa que hay razones preteóricas para pensar que el conocimiento no es una clase natural. En la medida en que la idea de que el conocimiento es un estado mental genuino tiene a esto último como corolario, esas razones preteóricas apuntan en contra de la propia (CEMf). Si esas razones preteóricas son adecuadas, entonces, contra Williamson, la tesis (CEMf) carece del apoyo prima facie que parecía tener. Se torna, de este modo, muy relevante evaluar en el plano teórico las virtudes de la caracterización del conocimiento como la actitud factiva estativa más general. Solo si dicha articulación sustantiva de (CEMf) representa ventajas teóricas es que podrán descartarse las razones preteóricas en contra de la misma.

Tras identificar las dos virtudes teóricas de la propuesta de Williamson como siendo (1) satisfacer una necesidad teórica surgida en el ámbito de la explicación causal de la acción y (2) explicar el valor del conocimiento, desarrollo la segunda parte de mi objeción. Para esto, presento argumentos de tipo lingüístico y filosófico que, empleando las herramientas mismas que Williamson usa a su favor, muestran la incapacidad de la teoría de satisfacer (1) y (2). Sin esto último, tampoco parece haber razones teóricas de peso para defender (CEMf). 
Cabe añadir que, dado que la propuesta de Williamson es quizás la más desarrollada en la literatura, la objeción que he presentado constituye un desafío más general a la posibilidad de comprender al conocimiento como un estado puramente mental.

\section{Bibliografía}

Austin, J. L. (1961), "Other Minds" en Philosophical Papers, Oxford, Oxford University Press.

Ayer, A. J. (1956), The Problem of Knowledge, Londres, MacMillan.

Cassam, Q. (2009), "Can the Concept of Knowledge be Analyzed?", en Greenough, P. y Pritchard, D. (eds.), Williamson on Knowledge, Oxford, Oxford University Press.

Chisholm, R. (1957), Perceiving: A Philosophical Study, Ithaca, Cornell University Press.

(1976), Theory of Knowledge, Nueva Jersey, Prentice Hall.

Clark, A. (2002), "Is seeing all it seems? Action, Reason and the grand illusion", Journal of Consciousness Studies, 9 (5-6), pp. 181-202.

Craig, E. (1990), Knowledge and the State of Nature: An Essay in Conceptual Synthesis, Oxford, Oxford University Press.

Dretske, F. (1969), Seeing and Knowing, Chicago, University of Chicago Press.

Foley, R. (2002), "Review: Knowledge \& its Limits", Mind, 111 (443), pp. 718-726.

Frenda, S. J., Knowles, E. D., Saletan, W., y Loftus, E. F. (2013), "False memories of fabricated political events", Journal of Experimental Social Psychology, 49 (2), pp. 280-286.

Fricker, E. (2009), "Is Knowing a State of Mind? The case against", en Greenough, P. y Pritchard, D. (eds.), Williamson on Knowledge, Oxford, Oxford University Press.

Girgus, J. S., Coren, S., y Fraenkel, R. (1975), "Levels of perceptual processing in the development of visual illusions", Developmental Psychology, 11 (3), pp. 268-273.

Greenough, P. y Pritchard, D. (eds.) (2009), Williamson on Knowledge, Oxford, Oxford University Press.

Gregory, R. L. (1968), "Perceptual Illusions and Brain Models", Proceedings of the Royal Society of London, Series B, Biological Sciences, 171 (1024), pp. 279-296.

Haslanger, S. (2006), "What Good Are Our Intuitions? Philosophical Analysis and Social Kinds", Proceedings of the Aristotelian Society, 
Supplementary Volumes, 80 (1), pp. 89-118.

Hazlett, A. (2010), "The Myth of Factive Verbs", Philosophy and Phenomenological Research, 80 (3), pp. 497-522.

Hellie, B. (2007), "Factive Phenomenal Characters", Philosophical Perspectives, 21, pp. 259-303.

Hoffman, D. (2011), "The construction of visual reality" en Blom, J. D, y Sommer, I. (eds.), Hallucination: Research and Practice, Springer.

Hoffman, D., Mark, J. y Marion, B. (2010), "Natural selection and veridical perception", Journal of Theoretical Biology, 266, pp. 504-515.

Kandel, E., Schwartz, J., y Jessell, T. (1995), Essentials of Neural Science and Behavior, Stamford, Appleton \& Lange.

Kitaoka, A., Gyoba, J. y Sakurai, K. (2006), "The visual phantom illusion: a perceptual product of surface completion depending on brightness and contrast", Progress in Brain Research, 154, pp. 247 262.

Kornblith, H. (2002), Knowledge and its place in nature, Oxford, Oxford University Press.

Kumar, V. (2014), "Knowledge as a natural kind term”, Synthese, 191 (3), pp. $439-457$.

Kusch, M. (2002), Knowledge by Agreement: The Programme of Communitarian Epistemology, Oxford, Oxford University Press.

Laroi, F. y Van der Linden, M. (2005), "Metacognitions in proneness towards hallucinations and delusions", Behavior Research and Therapy, 43, pp. 1425-1441.

Leite, A. (2005), "On Willliamson's Arguments that Knowledge is a Mental State", Ratio XVIII (2), pp.165-175.

Morrison, A. P. y Wells, A. (2000), "Cognitive factors in predisposition to auditory and visual hallucinations", British Journal of Clinical Psychology, 39, pp. 67-78.

Nagel, J. (2013), "Knowledge as a Mental State", Oxford Studies in Epistemology, 4, pp. 275-310.

Neta, R. y Rohrbaugh, G. (2004), "Luminosity and the Safety of Knowledge”, Pacific Philosophical Quarterly, 85, pp. 396-406.

Putnam, H. (1985), "Why Reason can't be naturalized", en Philosophical papers: Volume 3, Realism and Reason, Cambridge University Press.

Schacter, D. L., Guerin S. A. y St. Jacques, P. L. (2011), "Memory Distortion: an Adaptive Perspective", Trends in Cognitive Sciences, 15 (10), pp. 467-474.

Sellars, W. (1956), “Does Empirical Knowledge have a foundation?", en Sosa, E. y Kim, J. (eds.) (2000), Epistemology: an Anthology, 
Londres, Blackwell.

Shope, R. K. (1983), The analysis of Knowledge, Princeton, Princeton University Press.

Smithies, D. (2012), "Mentalism and Epistemic Transparency", Australasian Journal of Philosophy, 90 (4), pp. 723-741.

Stalnaker, R. (1974), "Pragmatic Presuppositions", en Munitz, M. y Unger, P. (eds.) Semantics and Philosophy, Nueva York, New York University Press.

Steup, M. (2009), “Are Mental States Luminous?” en Greenough, P. y Pritchard, D. (eds.), Williamson on Knowledge, Oxford, Oxford University Press.

Szpunar, K. K. y Schacter, D.L. (2013), "Get Real: Effects of Repeated Simulation and Emotion on the Perceived Plausibility of Future Experiences", Journal of Experimental Psychology: General, 142 (2), pp. 323-327.

Vendler, Z. (1967), Linguistics in Philosophy, Ithaca, Cornell University Press, caps. 1 y 4 .

Viger, C. (2006), "Is the Aim of Perception to Provide Accurate Representations? A Case for the "No" side", en Stainton, R. J. (ed.), Contemporary Debates in Cognitive Science, Malden, Blackwell.

Waring, J. D., Payne, J. D, Schacter, D. L. y Kensinger, E. A. (2010), "Impact of Individual Differences Upon Emotion-Induced Memory Trade-Offs", Cognition and Emotion, 24 (1), pp. 150-167.

Welbourne, M. (1993), The Community of Knowledge, Aldershot, Gregg Revivals.

Williams, M. (2007), "Why (Wittgensteinian) Contextualism is Not Relativism”, Episteme, 4 (1), pp. 93-114.

Williamson, T. (2000), Knowledge and its Limits, Nueva York, Oxford University Press.

(2005), "Precis of Knowledge \& its Limits" en Philosophy and Phenomenological Research, 70 (2), pp. 431-435.

(2007), The Philosophy of Philosophy, Malden, Blackwell. (2009) "Replies to critics" en Greenough, P. y Pritchard, D. (eds.), Williamson on Knowledge, Oxford, Oxford University Press.

Recibido el 8 de mayo de 2014; aceptado el 5 de noviembre de 2014. 